\title{
Reforma Sanitária e Informação em Saúde: Avaliação dos Registros Médicos em um Distrito Sanitário de Salvador, Bahia'
}

\section{Health Reform and Health Information: Evaluation of Medical Records in a Health District of Salvador, Bahia.}

\author{
Márcia G. Modesto ${ }^{2}$ \\ Esdras C. Moreira ${ }^{2}$ \\ Naomar de Almeida-Filho ${ }^{2}$
}

MODESTO, M. G.; MOREIRA, E. C. \& ALMEIDA-FILHO, N. de Health Reform and Health Information: Evaluation of Medical Records in a Health District of Salvador, Bahia. Cad. Saüde Pübl, Rio de Janeiro, 8 (1): 62-68, jan/mar, 1992.

This study aims at evaluating the quality of medical records in primary health services of the Health District of Itapagipe (Salvador/Bahia, Brazil), before and after the health reform of the Unified and Decentralized Health System (SUDS), initiated in 1987. Nine hundred and sixty one new records for 1986 and 850 for 1989 were evaluated with regard to: standardization of the record, legibility, and adequate entry of basic information from the medical history on the first visit. Comparing the pre - and post - health reform periods, improvement was observed in relation to standardization of records (from $49 \%$ to $94 \%$ ) as well as the recording of prescriptions and diagnoses (30\% of the records without diagnoses had prescriptions in 1986, dropping to 7\% in 1989). However, the proportion of legible records decreased slightly (from $76 \%$ to $71 \%$ ) and there was a marked increase in the frequency of blank records (from $6 \%$ to 19\%), indicating that, despite a better supply of material resources, there was not a corresponding progress in the quality of health information. Finally, even considering a marked improvement in the proportion of records with satisfactory quality (from 5 to 24\%), one can still find in 1989 a very high proportion (49\%) of records without registration of items from clinical examination.

Keywords: Health Services Epidemiology; Health-services Evaluation; Health Information

\section{INTRODUÇÃO}

Apesar do volume considerável de publicações sobre a proposta do Sistema Único de Saúde e sua implantação através da Reforma

\footnotetext{
'Trabalho realizado pelo Programa de Estudos Epidemiológicos e Sociais do Departamento de Medicina Preventiva da UFBA, com o patrocínio do CNPq (Proc. $\left.n^{\circ} 500150 / 88.5 \cdot M P\right)$. Uma versão preliminar foi apresentada no I Congresso Brasileiro de Epidemiologia, 4-6 de setembro de 1990.

${ }^{2}$ Departamento de Medicina Preventiva da Faculdade de Medicina da Universidade Federal da Bahia. Rua Padre Feijo, 29, $4^{9}$ andar, Anexo II, Salvador, BA, 40240, Brasil.
}

Sanitária (Paim, 1987; Teixeira, 1988; Cordei ro, 1989), observa-se uma escassez de estudos sobre as modificações determinadas por esse processo em setores especificos do sistema de saúde, tal como a área de registro e informação.

No processo de realização de um estudo de morbidade na atenção primária (Almeida Filho et al., 1991), constataram-se sérios problemas nos registros médicos da rede de serviços de saúde do Distrito Sanitário de Itapagipe, com elevado número de prontuários incompletos, ilegiveis ou mal-preenchidos. A partir desta observação, decidiu-se realizar uma análise mais aprofundada da questão, através de levan- 
tamento dos arquivos médicos das unidades de saúde da área, nos anos de 1986 e 1989, com o objetivo de acompanhar as alterações sofridas no sistema local de informação com o processo da reforma sanitária iniciado em 1987.

\section{MATERIAL E MÉTODOS}

Local da pesquisa: O Distrito Sanitário de Itapagipe localiza-se na região oeste de Salvador, sendo o primeiro com base na proposta do Sistema Unificado e Descentralizado de Saúde (SUDS). Com uma população de $145 \mathrm{mil}$ habitantes, compõe-se de 13 bairros, em sua maior parte de baixa renda e com pobres condições sanitárias e de habitação. Em 1986, existiam sete estabelecimentos públicos ou filantrópicos de Atenção Primária à Saúde (APS) na área: um centro médico-dentário municipal, dois postos e dois centros de saúde estaduais, um pequeno centro de saúde da Legião Brasileira de Assistência (LBA) e o ambulatório do Hospital Santo Antônio, um hospital de ensino de médio porte. Em 1989, um dos postos de saúde foi desativado e na época da coleta funcionava como abrigo para as vítimas da chuva que perderam suas casas.

Amostragem: No ano de 1986, houve 18.990 registros novos para a APS nos estabelecimentos de saúde da área. O centro da LBA, que no momento da coleta funcionava apenas com clínica pediátrica, e o centro médico-dentário municipal, que estava fechado para reformas, não foram incluídos na amostra. Os pacientes que recebiam cuidados secundários também não foram considerados. O ambulatório do Hospital Santo Antônio recebeu 14.609 pacientes para consulta em clínica médica, $77 \%$ da amostra total. Uma amostragem sistemática proporcional (ao contingente de registros de cada grupo de estabelecimentos de saúde) foi utilizada, com início aleatório em cada unidade, selecionando $1 / 30$ dos prontuários do ambulatório do Hospital Santo Antônio e 1/10 do pool de registros das outras unidades. A amostra final para o ano de 1986 foi composta de 961 indivíduos.

No período da segunda coleta, organizava-se um serviço de informática na área, só sendo possivel utilizar os prontuários do primeiro semestre de 1989, quando ocorreram 23.975 registros novos para APS nestas instituições. A amostragem foi igualmente do tipo sistemática proporcional, de início aleatório, selecionando 1/30 dos prontuários do ambulatório do Hospital Santo Antônio (HSA) e 1/25 do pool das outras unidades. A amostra final foi composta de 850 indivíduos. Em ambas as coletas, a maioria dos indivíduos foi do sexo feminino (60\%) e com idade abaixo de 35 anos (56\%), proporções superestimadas quando comparadas à população geral no local da pesquisa (IBGE, 1985).

Coleta de dados: Inicialmente, um estudo-piloto foi conduzido a fim de explorar as perspectivas da participação institucional, condições do arquivo médico disponivel e as possibilidades operacionais dos procedimentos de coleta. Os prontuários amostrados foram submetidos a um exame detalhado, seguindo procedimentos padronizados de avaliação. As informações sobre os tópicos em estudo foram cuidadosamente transferidas para um formulário especial, cobrindo oito seções: 1) Identificação; 2) Dados pessoais; 3) Características do cuidado dispensado; 4) Exame clínico (anamnese e exame físico); 5) Testes laboratoriais; 6) Informações sobre o diagnóstico; 7) Prescrição; 8) Avaliação da qualidade do preenchimento.

No processo de coleta, observou-se atentamente o preenchimento dos itens básicos da anamnese, como queixa principal e história da moléstia atual, o registro do exame físico, hipóteses diagnósticas e orientação terapêutica, a fïm de decifrar registros pouco legíveis e detectar ilegibilidade e ausência de informações nos prontuários amostrados.

Em 1990, época da segunda coleta de dados, houve uma modificação no serviço de matrícula e arquivo dos centros de saúde com a implantação de terminais de computadores. Foram criados três tipos de prontuários: 1) prontuário-familia, que inclui os registros dos individuos de uma mesma familia, residentes no Distrito em questão; 2) prontuário-individual; 3) ficha provisória, aberta para os individuos residentes fora da área, com direito a três consultas nas 
unidades de saúde pertencentes ao Distrito. Nesse novo sistema, todos os membros de uma família podem ser matriculados, adquirindo uma ficha com os seus dados pessoais preenchidos, apesar de não terem solicitado nenhuma consulta. Desta forma, os números de matrícula são somados aos números dos prontuários abertos por solicitação de consulta numa mesma lista para aquele período. Assim, a amostra retirada da lista de novas matrículas do primeiro semestre de 1989 continha um número elevado de prontuários em branco devido às fichas familiares.

Análise dos dados: Para uma melhor avaliação da qualidade do registro, foram analisados: 1) legibilidade dos registros; 2) padronização do prontuário; 3) preenchimento de itens indispensáveis da anamnese, como queixa principal, história da moléstia atual e exame físico. A legibilidade foi avaliada subjetivamente pelos coletores, que classificavam o prontuário como: legível, ilegível/parcialmente legivel. Foi considerada padronizada toda ficha de primeira consulta impressa em formulário timbrado, utilizada especificamente para o primeiro atendimento em cada serviço. Para o terceiro item acima mencionado, foi criada uma variável denominada "Avaliação do prontuário", que engloba a presença de registro de queixa, história da moléstia atual e exame físico. O prontuário foi classificado como "minimamente satisfatório" se constatados registros de pelo menos uma informação sobre cada um dos itens acima designados. $O$ exame físico foi considerado como realizado caso houvesse, pelo menos, o registro de um dado vital.
Empregou-se uma análise tabular comparativa dos dois períodos considerados. O Hospital Santo Antônio, por ser uma unidade de ensino, mereceu uma análise separada das outras unidades. No que diz respeito à conduta médi$\mathrm{ca}$, tomando como base os dados registrados nos prontuários, avaliou-se a relação entre o número de prontuários com diagnóstico registrado e a presença de prescrição nos mesmos. Dessa forma, foi possivel correlacionar os registros de diagnóstico e prescrição, com a finalidade de avaliar a conduta médica na APS com relação à capacidade de orientação terapêutica a partir de hipóteses diagnósticas. Os dados foram processados e analisados em microcomputador IBM-AT, empregando-se o software SPSS-PC (Nie et al., 1975).

\section{RESULTADOS}

A Tabela I revela, de forma global, uma redução nas proporções de prontuários legíveis e parcialmente legíveis e considerável aumento na proporção de prontuários em branco, quando comparamos os anos de 1986 e 1989. No que diz respeito aos prontuários legiveis, observamos que esta queda deveu-se, basicamente, ao grupo das outras unidades (68\% em 1986 e $58 \%$ em 1989), já que o HSA apresentou $86 \%$ de seus prontuários legíveis em 1986 e $88 \%$ em 1989. A ilegibilidade reduziu-se para ambos os grupos, apesar de ainda permanecer elevada nas outras unidades em 1989 (15\%). O considerável incremento na proporção de prontuários em branco (de 6\% para $19 \%$ em 1989) ocorreu não só às custas do grupo das

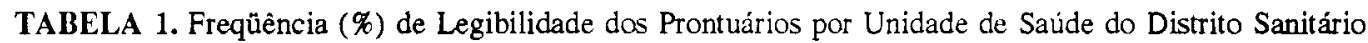
de Itapagipe, Salvador, 1986 e 1989

\begin{tabular}{lcccrrr}
\hline & \multicolumn{2}{c}{ Legível } & \multicolumn{2}{c}{ Ileg./P. Leg. } & \multicolumn{2}{c}{ Branco } \\
\hline Unidade & 1986 & 1989 & 1986 & 1989 & 1986 & 1989 \\
\hline H.S.A. & 85,6 & 88,0 & 11,5 & 4,2 & 2,9 & 7,8 \\
Outras Unidades & 67,8 & 57,6 & 23,4 & 14,8 & 8,8 & 27,6 \\
Total & 76,3 & 70,8 & 17,7 & 10,2 & 6,0 & 19,0 \\
\hline \hline
\end{tabular}


outras unidades, mas também do HSA, que passou de $3 \%$ de prontuários em branco em 1986 para $8 \%$ em 1989.

No que diz respeito à padronização do prontuário, existiam, em 1986, diferenças bastante expressivas entre os dois grupos em estudo. Foram considerados padronizados $83 \%$ dos prontuários do HSA e $18 \%$ dos prontuários das outras unidades. Em 1989, após a implantação do SUDS, $99,7 \%$ dos prontuários do HSA e $89 \%$ dos das outras unidades tinham sido preenchidos em folhas padronizadas, com formulários específicos para o registro de dados da primeira consulta (Tabela II).

A Tabela III revela que, no período em estudo, aumentou a proporção de prontuários considerados minimamente satisfatórios. Apesar do crescimento observado no grupo das outras unidades, ainda é bastante elevada a proporção (76\%) de prontuários que não possuem pelo menos os itens básicos de uma anamnese.

De acordo com a Tabela IV, os médicos do HSA prescreveram mais em 1989 (44\%) do que em 1986 (43\%); e a quantidade de prescrições ilegíveis decresceu de $5 \%$ para $1,7 \%$ neste periodo. As outras unidades apresentaram uma proporção menor de registro de prescrição e de prescrições ilegiveis em 1989. Em 1986, $44 \%$ dos prontuários tinham registro de prescrição, reduzindo-se para pouco mais de $30 \%$ em 1989.

TABELA 2. Freqũência (\%) de Prontuários Padronizados por Unidade de Saúde do Distrito Sanitário de Itapagipe, Salvador, 1986 e 1989

\begin{tabular}{llrrr}
\hline \hline & \multicolumn{3}{c}{ Padronização } \\
& \multicolumn{2}{c}{ Sim } & \multicolumn{2}{c}{ Não } \\
\hline Unidade & 1986 & 1989 & 1986 & 1989 \\
\hline H.S.A. & 82,5 & 99,7 & 17,5 & 0,3 \\
Outras Unidades & 18,0 & 88,7 & 82,0 & 11,3 \\
\hline Total & 48.6 & 94,1 & 51,4 & 5,9 \\
\hline \hline
\end{tabular}

TABELA 3. Avaliação Qualitativa dos Prontuários das Unidades de Saúde do Distrito Sanitário de Itapagipe, Salvador, 1986 e 1989

\begin{tabular}{lrrrr}
\hline \hline & \multicolumn{3}{c}{ Qualidade dos Prontuários } \\
& \multicolumn{2}{c}{ Satisfatório } & \multicolumn{2}{c}{ Não-Satisfatório } \\
\hline Unidade & 1986 & 1989 & 1986 & 1989 \\
\hline H.S.A. & 81,0 & 86,7 & 19,0 & 13,3 \\
Outras Unidades & 4,6 & 23,8 & 95,4 & 76,2 \\
\hline Total & 40,9 & 51,0 & 59,1 & 49,0 \\
\hline \hline
\end{tabular}

TABELA 4. Freqũência (\%) de Registro de Prescrição nos Prontuários das Unidades de Saúde do Distrito Sanitário de Itapagipe, Salvador, 1986 e 1989

\begin{tabular}{lcccccc}
\hline \hline & \multicolumn{2}{c}{ Ausente } & \multicolumn{2}{c}{$\begin{array}{c}\text { Prescrição } \\
\text { Presente }\end{array}$} & \multicolumn{2}{c}{ Ilegível } \\
\hline Unidade & 1986 & 1989 & 1986 & 1989 & 1986 & 1989 \\
\hline H.S.A. & 52,7 & 54,4 & 42,7 & 43,9 & 4,6 & 1,7 \\
Outras Unidades & 41,8 & 58,6 & 43,6 & 33,7 & 14,6 & 7,7 \\
\hline Total & 44,3 & 55,6 & 43,4 & 39,7 & 12,3 & 4,7 \\
\hline \hline
\end{tabular}


A Tabela $\mathrm{V}$ correlaciona os registros de diagnóstico e prescrição, mostrando que, em 1986 , nas outras unidades, $51 \%$ dos prontuários sem registro de diagnóstico apresentavam registro de prescrição. Este percentual caiu, em 1989, para $13 \%$. No HSA, $8 \%$ em 1986 e $2 \%$ em 1989 não apresentavam diagnóstico, apesar do registro de prescrição. Ainda no HSA, $46 \%$ dos prontuários continham registro de diagnóstico, mas não de prescrições, em 1986, com um discreto aumento (49\%) em 1989. Para as outras unidades, foi marcante o aumento na proporção de prontuários com registro de diagnóstico, de 9\% em 1986 para 64\% em 1989.

\section{DISCUSSÃO}

Após a implantação da Reforma Sanitária, observou-se uma melhoria significativa em relação à padronização dos registros médicos utilizados no Distrito Sanitário de Itapagipe. Aproximadamente $94 \%$ dos prontuários incluiam formulários com campos adequados ao preenchimento pelo profissional de saúde. Porém, apesar da melhoria no fornecimento de material, o preenchimento ainda é deficiente. A proporção de prontuários legíveis diminuiu e a de prontuários em branco foi bem mais elevada em 1989, quando comparadas com 1986. Com relação aos prontuários em branco, as elevadas proporções (28\%) para o grupo das outras unidades já eram esperadas, pois, com a mudança do sistema de matrícula, foram abertos prontuários sem que houvesse pedido de consulta. Tais modificações no sistema de matrícu- las não ocorreram no Hospital Santo Antônio, porém, aí, a proporção de prontuários em branco também cresceu em 1989. Estes resultados aproximam-se aos encontrados em recente avaliação dos serviços de saúde em Cáceres, MT (Lotufo \& Duarte, 1987), onde cerca de $20 \%$ dos registros hospitalares encontravam-se sem diagnóstico ou com diagnósticos ilegíveis ou incoerentes.

Entretanto, em relação à qualidade dos registros, é alarmante a proporção de prontuários considerados não-satisfatórios (quase 50\%), por não apresentarem pelo menos os itens básicos da anamnese de primeira consulta. Estes dados devem-se, essencialmente, aos prontuários do grupo das outras unidades, que englobam os centros e postos da rede pública de APS do Distrito em estudo. Isto provavelmente reflete a baixa qualidade do atendimento que é oferecido à população por profissionais com baixa remuneração, tempo parcial no serviço e vinculação simultânea com o setor privado. É importante enfatizar, entretanto, que, com relação aos dados de 1986, houve uma queda na proporção de prontuários não-satisfatórios, o que pode indicar discreta melhoria no cuidado com o preenchimento dos registros.

No que diz respeito ao registro de prescrição e diagnóstico, em 1989, o corpo clínico das outras unidades prescreveu bem menos que em 1986, ao mesmo tempo em que passou a diagnosticar ou registrar suas suspeitas diagnósticas de forma mais intensa. Em 1986, a proporção de prontuários com registro de prescrição, porém sem diagnóstico, alcançou um percentual de quase $51 \%$. Em 1989, esta proporção caiu para $13 \%$. No HSA, apesar de ter aumen-

TABELA 5. Frequêencia (\%) de Registro de Diagnóstico e Prescrição por Unidade de Saúde do Distrito Sanitário de Itapagipe, Salvador, 1986 e 1989

\begin{tabular}{|c|c|c|c|c|c|c|c|c|}
\hline \multirow[b]{3}{*}{ Unidade } & \multicolumn{8}{|c|}{ Diagnóstico e Prescrição } \\
\hline & \multicolumn{2}{|c|}{ S/D S/P } & $\mathrm{S} / \mathrm{D}$ & $\mathrm{C} / \mathrm{P}$ & \multicolumn{2}{|c|}{$\mathrm{C} / \mathrm{D} \quad \mathrm{S} / \mathrm{P}$} & $\mathrm{C} / \mathrm{D}$ & $\mathrm{C} / \mathrm{P}$ \\
\hline & 1986 & 1989 & 1986 & 1989 & 1986 & 1989 & 1986 & 1989 \\
\hline H.S.A. & 7,1 & 5,6 & 7,6 & 2,0 & 45,5 & 48,7 & 39,8 & 43,7 \\
\hline Outras Unidades & 40,0 & 23,7 & 50,6 & 12,6 & 2,8 & 35,0 & 6,6 & 28,7 \\
\hline Total & 23,8 & 14,7 & 30,2 & 7,4 & 23,1 & 41,8 & 22,9 & 36,1 \\
\hline
\end{tabular}


tado a proporção de registros de prescrição, quando se associa a presença de diagnóstico e prescrição, observa-se que a maioria dos prontuários apresentava diagnóstico sem prescrição, seguida daqueles que continham tanto diagnóstico quanto prescrição.

Em síntese, os resultados do presente estudo revelam que, mesmo após a implantação do SUDS, os médicos envolvidos na APS continuam preenchendo insatisfatoriamente os prontuários, apesar de se registrar maior volume de diagnósticos e menor prescrição de medicamentos. Provavelmente, a organização recém-implementada nestes serviços, com o fornecimento de fichas padronizadas, junto com a informatização do processo de matrícula e arquivos, vem contribuindo para a construção de uma infra-estrutura mais eficiente. Porém, ainda é necessário rever as dificuldades relacionadas aos recursos humanos, que necessitam com urgência valorizar adequadamente o papel da informação em saúde na consolidação de um sistema de assistência efetivamente capaz de atender às necessidades da população.

Vale ressaltar, no entanto, que o Distrito estudado não é representativo da realidade mais comum dos serviços na era do SUDS, e sim de um processo local específico de mudança, onde procurou-se implantar, de forma mais comprometida, as idéias da reforma sanitária. De todo modo, com o presente trabalho, esperamos ter contribuido de forma direta para a discussão e formulação de programas locais de saúde, visando a melhoria do preenchimento dos registros médicos que, afinal, refletem, em última instância, a qualidade do atendimento prestado à população.

\section{AGRADECIMENTOS}

Os autores agradecem ao Prof. Jairnilson Paim, por suas críticas e comentários a uma versão preliminar, bem como a um anônimo revisor do CSP, que muito contribuiu para o rigor deste trabalho e para a clareza do texto final.

\section{RESUMO}

MODESTO, M. G.; MOREIRA, E. C. \& ALMEIDA-FILHO, N. de Reforma

Sanitária e Informação em Saúde:

Avaliação dos Registros Médicos em um

Distrito Sanitário de Salvador, Bahia. Cad.

Saúde Públ., Rio de Janeiro, 8 (1): 62-68, jan/abr, 1992.

O presente estudo objetivou avaliar a qualidade dos registros médicos dos serviços de atenção primária à saúde no Distrito Sanitário de Itapagipe, em Salvador/Bahia, Brasil, antes e depois da reforma sanitária do Sistema Unificado e Descentralizado de Saúde (SUDS), iniciada em 1987. Foram analisados 961 prontuários novos para o ano de 1986 e 850 para o ano de 1989 , em relação aos seguintes aspectos: padronização do prontuário, legibilidade e preenchimento dos itens básicos de uma anamnese de primeira consulta. Comparando-se os períodos pré e pós-reforma sanitária, observou-se uma melhoria em relação à padronização dos prontuários (de $49 \%$ para $94 \%$ ) e ao registro de prescrição e de diagnósticos (30\% dos prontuários sem registro de diagnóstico apresentavam registro de prescrição em 1986, passando a 7\% em 1989). Entretanto, ocorreu um decréscimo na proporção de prontuários legiveis (de $76 \%$ para $71 \%$ ) e um aumento importante na freqüência de prontuários em branco (de $6 \%$ para $19 \%$ ), indicando que, apesar da melhoria no suprimento de recursos materiais, não se observou um avanço correspondente na qualidade da informação em saúde.

Finalmente, apesar de uma acentuada melhoria na proporção de prontuários com qualidade satisfatória (de $5 \%$ para $24 \%$ ), ainda se observou uma altíssima proporção (49\%) de prontuários sem registro dos itens de exame físico.

Palavras Chave: Epidemiologia de Serviços de Saúde; Avaliação de Serviços de Saúde; Informação em Saúde 


\section{REFERÊNCIAS BIBLIOGRÁFICAS}

ALMEIDA FILHO, N. M.; SANTANA, V.; OLIVEIRA, M. B.; MOREIRA, E. C. \& MODESTO, M. G., 1991. Mental Disorders in Primary Care in a Health District of Bahia, Brazil. In: Primary Care and Psychiatric Epidemiology (B. Cooper \& M. Eastwood, eds.), pp. 133-141, London: Routledge.

BAHIA-SECRETARIA DE SAÚDE, 1987. Sistema Unificado e Descentralizado de Saúde no Estado da Bahia. Salvador: Secretaria de Saúde.

CAMPOS, G. W. S., 1988. A reforma sanitária necessária. In: Reforma Sanitária: Itália e Brasil (G. Berlinguer; S. F. Teixeira \& G. W. S. Campos), pp. 179-194, São Paulo: Hucitec.

CORDEIRO, H., s/d. A Reforma Sanitária; Bases estratégicas e operacionais para a descentralização e unificação do sistema de saúde. Rio de Janeiro: Inamps (Mimeo.).

INSTITUTO BRASILEIRO DE GEOGRAFIA E ESTATISTICA (IBGE), 1985. Censo Demográfico, 1980. Rio de Janeiro: Fundação IBGE.

LOTUFO, M. \& DUARTE, E. C., 1987. Avaliação dos serviços de saúde do município de Cáceres, MT (Brasil): Contribuiçōes e programação local. Revista de Saúde Pública, 21: 427-438.

MENDES, E. V., 1987. O Sistema Unificado e Descentralizado de Saúde no Atual Contexto da Reforma Sanitária Brasileira. Conferência proferida no Seminário Interinstitucional sobre a Reforma Sanitária e a Organização dos Serviços de Saúde no Rio Grande do Norte, Natal.

NIE, N. H.; HULL, H.; JENKINS, J. G.; BENT, D. H. \& STEINBUNNER, K., 1979. Statistical Package For the Social Sciences. New York: McGraw-Hill.

PAIM, J., 1987. Sistema Unificado e Descentralizado de Saúde - Suds/Bahia (Sesab/Inamps/Ufba/ Sucam/FSESP) Governo Democrático da Bahia. O que é Reforma Sanitária? Revista Baiana de Saúde Pública, 14: 232-243.

TEIXEIRA, C. F., 1988. Reforma Sanitária/Suds/Sus/ Distritos Sanitários, a equação da mudança da saúde na Bahia. Salvador: DMP/Universidade Federal da Bahia (Mimeo.). 